\title{
Auditory Neuropathy Spectrum Disorder-A Study In Nicu High Risk Infants
}

\author{
Dr. Manish Joshi ${ }^{1}$,Dr.(Mrs.) Swagata \\ Khanna $^{2}$,Dr.(Mrs.)H.Bora ${ }^{3}$,Dr.A.K.Biswas ${ }^{4}$,Dr.G.K.Pathak \\ ${ }^{I}$ (Postgraduate Trainee, Dept Of Otorhinolaryngology And Head \& Neck Surgery, Guwahati Medical College) \\ ${ }_{2}^{2}$ (Professor,Dept Of Otorhinolaryngology And Head \& Neck Surgery, Guwahati Medical College) \\ ${ }_{3}^{3}$ (Associate Professor, Dept Of Otorhinolaryngology And Head \& Neck Surgery, Guwahati Medical College) \\ ${ }^{4}$ (Lecturer Audiology, Dept Of Otorhinolaryngology And Head \& Neck Surgery, Guwahati Medical College) \\ 5 (Professor And Head, Dept Of Otorhinolaryngology And Head \& Neck Surgery,Guwahati Medical College)
}

\begin{abstract}
Auditory neuropathy spectrum disorder (ANSD) is a condition of multifactorial origin in which transmission of sound to the brain is abnormal. Children who suffer from this condition experience difficulties with speech perception, especially in noise, and the development of language skills.

This study is intended to determine the prevalence and audiological profile of auditory neuropathy spectrum disorder in NICU high risk infants as defined by joint commission of infant hearing(JCIH 2007).

A prospective study of one year duration was conducted from $1^{\text {st }}$ january 2015 to $31^{\text {st }}$ December 2016 in 100 number of NICU infants with high risk factors at GMCH,assam.

All infants were subjected to history and audiological evaluation.Diagnosis of ANSD was made with normal Otoacoustic emissions but abnormal brain stem evoked response. Finaly prevalence and audiological profile was determined.
\end{abstract}

Keywords: Auditory neuropathy spectrum disorder,Prevalence, Nicu infants

\section{Introduction}

The term auditory neuropathy is a relatively recent clinical diagnosis due to dysfunction of the synapse of the inner hair cells and auditory nerve and/or auditory nerve itself,was origionaly proposed by Starr and colleagues(Starr et al.1996)[1].It is characterized by evidence of normal cochlear hair cell function(preservation of otoacoustic emissions and cochlear microphonics),and abnormal auditory pathway function starting from eighth nerve.Some investigators(Berlin et al.2001,2003;Rapin \&Gravel,2006)have expressed dissatisfaction with the term 'auditory neuropathy' as constellation of test results defining this disorder doesnot provide direct evidence of auditory nerve dysfunction or 'neuropathy'.Indeed, only a subset of individuals with this disorder will be found to have abnormal auditory nerve function.Other lesions,for example,mutation of otoferlin gene(OTOF) which results in synaptic dysfunction at the junction of inner hair cell/auditory nerve, will produce the same constellation of auditory test results in affected individuals(Yasunaga et al.,1999; Yasunaga et al.2000)to address this and other concerns berlin and colleagues[2] proposed the term 'auditory dyssynchrony'. And the most recent nomenclature is 'auditory neuropathy spectrum disorder'(ANSD).The term 'spectrum' was used ,because expression of this disorder in everyday hearing and communication encompasses a spectrum ranging from limited or mild effects(complains of difficulty hearing in 'noisy' listening conditions)to profound effects(inability to 'hear' in any listening conditions, functionally 'deaf'), and also to include site of lesion other than auditory nerve.

Ansd is a unique disorder which affects speech and language development inpediatric population by impairing normal hearing mechanism,thus,results in poor speech discrimination.It has been shown that this disorder affects an individual's ability to process rapidly changing acoustic signals,known as auditory temporal processing, which results in normal to severly impaired speech detection and pure tone thresholds. The range of functional hearing abilities in individuals with auditory neuropathy is vast.Some individuals experience little or no difficulty hearing and understanding despite abnormal auditory test results, others complains of hearing but not understanding,specialy in background noise.Some individual demonstrate fluctuant hearing abilities ,reporting 'good hearing days'and 'bad hearing days'.Finally,some children and adults are functionally deaf.

Starr et al. (2004) suggested segmenting the disorder in two types-type 1 (presynaptic) and type 2(post synaptic).In 2008, he further refined the terminology by site of disorder .If the auditory nerve was involved but the inner hair cells and synapses were spared he classified the disorder as 'auditory synaptic disorder' and if the inner hair cells or their synapses were involved with sparing of auditory nerve term 'auditory synaptic disorder'was used. 


\section{Aims And Objective}

To determine the prevalence and audiological profile of auditory neuropathy spectrum disorder (ANSD) among NICU high risk infants.

\section{Materials And Methods}

A prospective study of one year duration was conducted from $1^{\text {st }}$ january 2015 to $31^{\text {st }}$ december 2016 ,in 100 number of NICU infants with high risk factors(age group 0-1year) as defined by joint committee on infant hearing $(\mathrm{JCIH}) 2007$. High risk infants had at leastsone of risk factors-

1. Birth weight less than $1500 \mathrm{gm}$.

2. Craniofacial abnormality.

3. Suffering from bacterial meningitis.

4. Intrauterine infection like measles, syphilis, toxoplasmosis, CMV and herpes.

5. Put on ototoxic drugs during neonatal period.

6. Put on ototoxic drugs during pregnancy

7. APGAR score of 0-4 in first minute or of 0-6 in fifth minute.

8. Prematurity (gestational age lower than 37 week).

9. Hyperbiliruinemia requiring blood exchange.

10. Any other potential risk factor.

The selection criteria used for diagnosis of ANSD was normal or near normal cochlear hair cells function (preservation of otoacoustic emission and/or cochlear microphonics) and absent or abnormal auditory nerve functions (absent or severely abnormal auditory brainstem potential).

First of all NICU infants which are referred to ENT outpatient department were assessed for high risk factors for hearing loss,biographical information was collected from hospitalrecords, and infants were labelled as high risk category (as defined by JCIH position statement 2007) . Complete audiological test battery were readministered on these children to diagnose auditory neuropathy.

Audiological test battery include-

\section{Audiological testing}

1) Otoscopic examination 2)acoustic immitance measures of middle ear function.

3) Behavioural assessment of puretonethresholds.

4) Distortion product otoacoustic emissions

5) Diagnostic auditory brainstem response-

\section{Procedure-}

The interacoustics impedance A766 was used to record immitance measures in this study.It includes Yadmittance tympanograms and acoustic reflexes. Behavioural assessment of puretone thresholds using developmentally appropriate,conditioned test procedures(visual reinforcement audiometry or conditioned orientation reflex audiometry).For very young or developmentally delayed infants,behavioural observation audiometry was used to observe the infant's response to sound. In evoked oto- acoustic emissions (OAEs), normal distortion product otoacoustic emissions (DPOAEs)was considered as a response with amplification above the background noise level of $3 \mathrm{~dB}$ in the octave band frequency. Auditory brainstem evoked potentials were recorded in two channel setup with four electrode configuration, that is, two electrode on both sided mastoid processes, one electrode on fore head as ground point and one at vertex to optimize detection of wave V. Band pass from 30 or $100 \mathrm{~Hz}$ to $4000 \mathrm{~Hz}$ was used to detect principle ABR waves. Click stimuli (rare fraction) were presented monaurally on repetition at rate 11.1 to $33.3 / \mathrm{s}$ and at intensities from $55 \mathrm{dBnHL}$ to 85 $\mathrm{dBnHL}$ and in most cases,95dBnHL.both condensation and rarefaction clicks were used in order to monitor for cochlear microphonics. Repeated wave replicability testing was administered to cross-check the ABR wave forms. Different averages were documented for wave interpretation throughout testing. Electrode generated impedance value and artifact rate were eventually monitored to maintain test-retest reliability.

\section{Results}

Out of 100 studiesstudied sensorineural hearing loss was detected in 22 infants.

\begin{tabular}{|l|l|l|}
\hline Subjects assesed & Sensorineural hearing loss & prevalence \\
\hline 100 & 22 & $22 \%$ \\
\hline
\end{tabular}

Most common risk factor was hyperbilirubinemia(n-54) and it was most commonlyassociated with sensorineural hearing loss(n-12). 


\begin{tabular}{|l|l|l|}
\hline Risk factor & subjects & Sensorineural hearing loss \\
\hline Hyperbillirubinemia & 54 & 12 \\
\hline Ototoxic medications & 38 & 6 \\
\hline Hypoxic ischemic encephalopathy & 27 & 5 \\
\hline Preterm birth & 24 & 7 \\
\hline Intrauterine infections & 18 & 1 \\
\hline Ear deformities & 1 & 1 \\
\hline Hereditary hearing loss & 1 & 0 \\
\hline
\end{tabular}

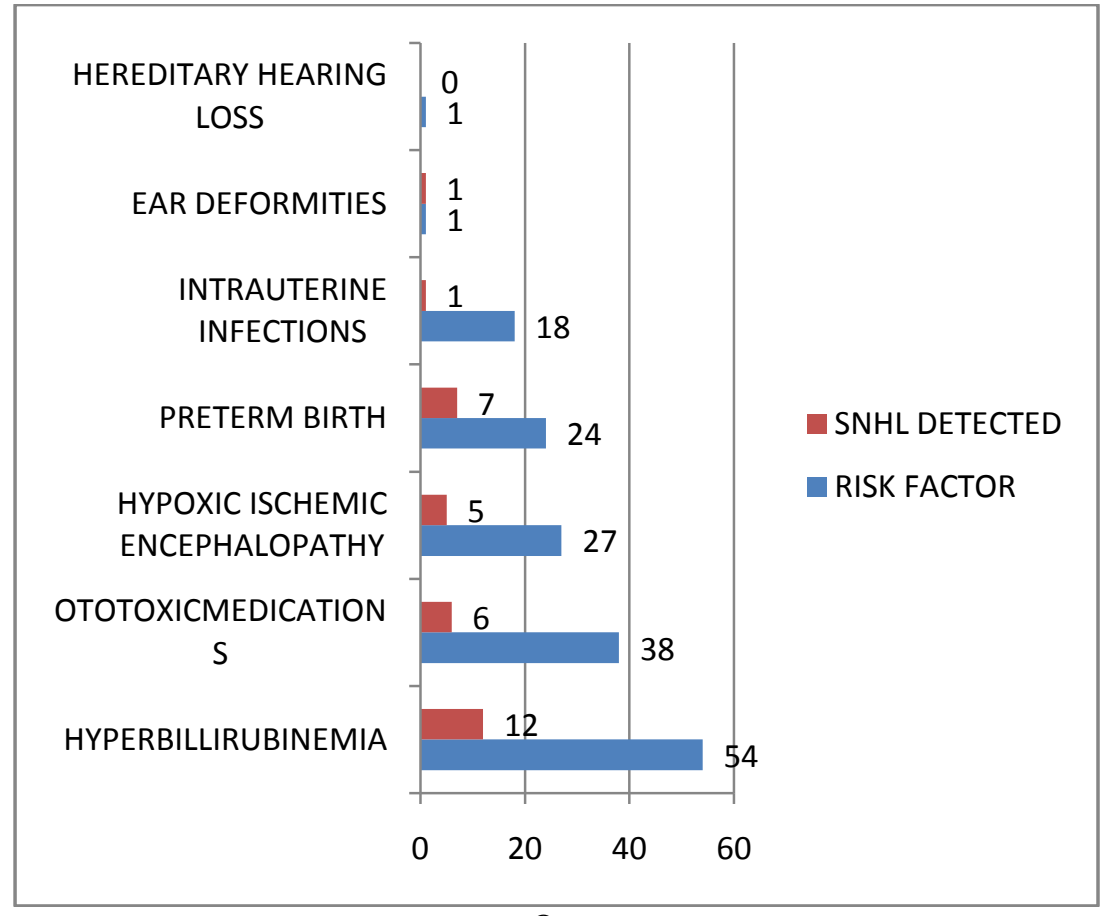

Out

Out of these 22 cases with sensorineural hearing loss, 2(9.09\%) showed significant diagnostic characteristics of ANSD ,such as preservation of cochlear microphonics, absent ABR and elevated MEMRs, poor speech recognition scores and elevated audiometric/free-field/BOA thresholds. Out of $2(50 \%, n=1$ was male and $50 \%, \mathrm{n}=1$ was female). Regarding to the etiological factors and clinical history, female patient had history of prematurity . Male infant gave history of mechanical ventilation and hyperbillirubinemia.

\section{Disscussion}

Prevalence of ANSD varies in different studies .Stein et al.(1996)in their study of 100 subjects in special care nursery found prevalence of 4\%,whereas Psarommatis et al.(1997)[3]( icu set up) and Rance et al.(1999)[4]('at risk infants') found the prevalence to be $1.96 \%$ and $0.23 \%$ respectively.Bielecki et al.(2012) in his study found that out of 352 children with SNHL18(5.1\%)were diagnosed with ANSD.In our study out of 22 infants with SNHL, 2(9.09\%)were diagnosed to have ANSD.

The etiology of ANSD seems to be multifactorial.the most commonly reported neonatal conditions associated are anoxia andhyperbilirubinemia.Starr et al.(2000)estimated that postviralinfectous processes were involved in 10\% of the 67 patientsfrom their database .Specific etiologic details were not presented ,but other studies have reported that mumps and meningitis can be associated with the ANSD.Among the proposed cause in children are genetic disorder,which may include mutation of otoferlin (OTOF)gene(Yasunaga et al.,1999; Yasunaga et al.2000) [5][6].Hereditary motor and sensory neuropathies such as Charcot-Marie-Tooth Syndrome make up a relatively high proportion of the adult ANSD cases.In our study ANSD was associated with mechanical ventilation and hyperbilirubinemia and prematurity.

Middle ear muscle reflexes(acoustic reflexes)are absent in individuals with $\operatorname{ANSD(Berlin~et~}$ al.2005)[7].Because normative data on acoustic reflex thresholdsin very young infants using high probe tone frequency $(1000 \mathrm{hz})$ have not been yet established,this procedure is not required to diagnose ANSD.Nevertheless, a complete test battery for ANSD should include middle ear muscle reflex testing whenever possible. Suppression of otoacoustic emissions by contralatralnoise is abnormal in individuals with ANSD(Hood et al.,2003).Although this test has not gained widespread clinical usage, it is a potential candidate for further diagnostic studies in individuals with reliably recorded OAEs. 
In newborns and very young infants measurements of OAEs may be compromised by presence of residual fluid in the ear canal/middle ear(Doyle et al.2000)[8] or otitis media with effusion(OME).OAEs may present initially and disappear over time in individuals with ANSD(Starr et al.,1996).Loss of OAEs, however,doesnot reflect change in auditory function or signal conversion of ANSD to sensorineural hearing loss.Cochlearmicrophonics(CMs)also provide a valid measure of hair cell function.CMs,remain present in an individual with ANSDdespite loss of OAEs(Starr et al.,1996).CMsare easily recorded from standard ABR recording protocols when insert earphones are used(Berlin et al.,2003;Starr et al.2001).Stimulus artifact precludes effective recording of CMs when electromagnetic circumaural earphones are used.(Berlin et al.2003; Stone et al.1986[9]).

Caution should be used in interpretation of results when these tests are used in infants below 36 weeks gestational age.Repetaedmeasures,over several weeks or months, are recommendedto determine the reliability of test results.Because 'transient'ANSD has been reported in some infants (Attias\& Raveh,2007;Madden et al.2002;)[10][11],particularly those with hyperbilirubinemia,hypoxia,ischemia and central nervous system immaturity(Attias\&Raveh,2007).Therefore frequent monitoring by the ANSD test battery is recommended to establish the stability of test results,Specially in the first two years of life.

There are three principal reason for infants with ANSD to receive comprehensive medical,developmental and communication assessment .First,defining etiology for predicting if the condition may be transient or permanent,determining if medical or surgical treatment is needed and answering parents questions about cause of their infants hearing disorder.Second,because infants with ANSD,Specially those who received care in the NICU, are at risk for additional disabilities,early identification of developmental delay is important for optimum child development.Third,infants with ANSDmay develop additional central or peripheral neuropathies secondary to specific diagnosis(Starr et al.1996).

ANSD may be unilateral or bilateral.The possibility of cochlear nerve deficiency(absent or small cochlear nerve) should be considered for all children with ANSD and specially in well babies with no significant history related to ANSD(Buchman et al.2006)[12]or infants with unilateral craniofacial anomalies(Carvalho et al.1999)[13].

Temporal processing or encoding the temporal characteristics of speech,is affected in subjects with ANSD(Zeng et al.,2005[14];Rance et al.2004)resulting in a disproportionate loss in speech understanding ability relative to the individual's pure tone thresholds.(Rance et al.1999; Rance et al.2002;Starr et al.1999[15]).Although conventional hearing aids improve sound audibility, they do not resolve temporal processing deficits.Therefore children with ANSD may not experience the same benefits from hearing aids expected from children with typical SNHL in whom temporal processing is relatively unaffected.Trial use of an FM system,specially in structured and spontaneous language-learning activities,should also be considered for children with ANSD.

Despite an adequate trial with appropriately fitted amplification ,some children with ANSD may demonstrate poor prognosis in speech understanding ability and auditory language development.For these children cochlear implantation should be considered,regardless of behavioural audiometric thresholds .Cochlear implantation offer the possibility of improving auditory temporal processing by stimulating synchronous discharge of the auditory nerve.ABR, which requires neural synchrony,can be electrically evoked in many individuals with cochlear implants(Peterson et al.2003;Shallop et al.2003)[16].

Counselling families of infants and young children with ANSD is one of the greatest challenges associated with this disorder. Because developmental effects of ANSD cannot be predicted from test results obtained in the earliest months or even years of life ,families struggle with the uncertainity of what diagnosis means relative to their infants growth and development.The significance of ANSD diagnosis may be difficult for families to appreciate as they struggle to understand their infants complex medical and developmental needs.Strongsupport system,including parents of children with similar diagnosis and professionals with expertise in clinical social work and family counselling, should be available to meet the ongoing and changing needs of families.

\section{Conclusion}

Audiological management and speech and language intervention for infants and young children with auditory neuropathy spectrum disorder is challenging.Because the range of functional hearing ability in this disorder is so great,each child with the diagnosis is unique.Furthermore the developmental consequences cannot be predicted on the basis of auditory test results obtained in infants,guidelines that exist for identification and management of infants and young children with 'typical'sensorineural hearing loss(SNHL)do not entirely fit the special needs of infants with auditory neuropathy. All suchchildren need to be reviewed and monitored in a similar way, and their management differs from that of children with conventional sensorineural or conductive hearing loss in important ways.Children with ANSD can develop into healthy and dynamic citizens with happy 
personal life ,successful academic experiences,and satisfying careers .Clinicians should help families realize this goal by identifying and supporting the unique strengths and abilities of the child and family.

\section{References}

[1]. Starr, A.; Picton, T. W.; Sininger, Y. S.; Hood, L.J.;Berlin, C. I. Auditory neuropathy. Brain, 119, pp. 741 -

[2]. 753, 1996

[3]. Berlin ,C.1,Hood,L.j,Rose K.(2001).On renaming auditory neuropathy as auditory dyssynchrony.Audiol Today13:15-17

[4]. PsarommatisIM,TsakanikosMD,KontorgianniAD,et al.(1997).Profoundhearing loss and presence of click evoked otoacousticemissionsin the neonate:A report of two cases.Int J Pedotorhinolaryngol 31:237-243

[5]. Rance G, Beer DE, Cone-Wesson B, Shepherd RK, Dowell RC, King AM, et al. Clinical findings for a group of infants and young children with auditory neuropathy.Ear Hear. 1999 20: 238 - 252

[6]. Varga, R.; Kelley, P. M.; Keats, B. J.; Starr, A.; Leal, S. M.; Cohn, E.; Kimberling, W. J. Non-syndromic

[7]. recessive auditory neuropathy is the resultof mutations in the otoferlin (OTOF) gene. J Med Genet, 40, pp. 45-50,2003.

[8]. Yasunaga,S.,Grati.,M.,Cohen-Salmon,M.,El-Amraoui,A.,Mustapha,M.,Salem,N.,Petit,C.,(1999).A mutation OTOF,encodingotoferlin, a FER-1 like protein,causes DFNB9, a nonsyndromic form of deafness.Nature Genetics,21,363-369.

[9]. Berlin CI, Hood LJ, Morlet T, Wilensky D, St John P, Montgomery E, Thibodaux M(2005), Absent or elevated middle ear muscle reflexes in the presence of normal otoacoustic emissions: a universal finding in 136 cases of auditory neuropathy/dys-synchrony.J Am AcadAudiol. 2005 Sep;16(8):546-53.

[10]. Doyle,K.J.,Rodgers,P.Fujukawa,S.,\&Newman,E.(2000).External and middle ear effects on infants hearing screening test results.Archives of Otolaryngology-Head \& Neck Surgury,122(4),477-481.

[11]. Stone,J.L.,Hughes,J.R.,Kumar,A.,Meyer,D.,Subramanian,K.S.,Zalkind,M.S.,\&Fino,J.(1986)Electocochleography recorded noninvasively from the external ear.Electroencephalography and Clinical Neurophysiology,63,494-496.

[12]. Attias,J.,Muller4,N.,Rubel,Y.,\&Raveh,E.(2006).Multiple auditory steady-state responses in children and adults with normal hearing,sensorineural hearing loss,or auditory neuropathy.Annals of Otology,Rhinology,and Laryngology,115(4),268-276.

[13]. Madden C, Rutter M, Hilbert L, Greinwald JH Jr, Choo DI. Clinical and audiological features in auditory

[14]. neuropathy. Arch Otolaryngol Head Neck Surg. 2002;128(9):1026-30.

[15]. Buchman,C.A.,Roush,P.A.,Teagle,H.F.F.,Brown,C.J.,Zdanski,C.I.,\&Grose,J.H.(2006).Auditory neuropathy characteristics in children with cochlear nerve deficiency.Ear and hearing,27(4),399-408.

[16]. Carvalho,G.J.,Song,C.S.,Vargervik,K.,\&Lalwani,A.K.(1999).Auditory and facial nerve dysfunction in patients with hemifacialmicrosmia.Archives of Otorhinolaryngology-Head \& Neck Surgury, 125(2),209-212.

[17]. Zeng,F.G.,Kong,Y.Y.,Michalewski,H.J.,\&Starr,A.(2005)Perceptual consequences of disrupted auditory nerve activity.Journal of Neurophysiology,93(60,3050-3063

[18]. Zeng, F. G.; Oba, S.; Garde, S.; Sininger, Y.; Starr A.Temporal and speech processing deficits in auditory

[19]. neuropathy. Neuroreport, 10, pp. 3429-3435, 1999

[20]. Peterson,A.,Shallop,J.,Driscoll,C.,Breneman,A.,Babb,J.,Stoeckel,R.,\&Fabry,L.(2003).Outcomes of cochlear implantation in children with auditory neuropathy.Journal of American Academy of Audiology,14(4),188-201. 